\title{
Thirty-day readmission rate and discharge status following total hip arthroplasty using the supercapsular percutaneously-assisted total hip surgical technique
}

\author{
Wade Gofton • James Chow • K. Dean Olsen • \\ David A. Fitch \\ Received: 25 October 2014 / Accepted: 27 October 2014 /Published online: 16 November 2014 \\ (C) The Author(s) 2014. This article is published with open access at Springerlink.com
}

\begin{abstract}
Purpose Recent studies have reported nearly $40 \%$ of costs associated with a 30-day episode-of-care for total joint replacements are due to post-discharge activities and $81 \%$ of those are specifically due to unplanned readmissions and discharging patients to post-acute care facilities. The purpose of this study was to determine these two key variables for total hip arthroplasty (THA) patients implanted using a tissuesparing surgical technique and to see how these values compare to those previously reported in the United States.

Methods The healthcare databases at three institutions were searched for primary THA patients implanted using the supercapsular percutaneously-assisted total hip (SuperPath) surgical technique between January 2013 and July 2014. Data elements included 30-day all-cause readmission rate, discharge status, transfusion rate, complications, and length of stay (LOS).

Results Data were available for 479 THAs. The 30-day allcause readmission rate, transfusion rate, and average LOS was $2.3,3.3 \%$, and 1.6 days, respectively. Over $91 \%$ of patients were discharged routinely home, $4.1 \%$ to skilled nursing facilities, $3.8 \%$ to home health care, and $0.6 \%$ to inpatient
\end{abstract}

\section{W. Gofton}

Ottawa Hospital - Civic Campus, J153-1053 Carling Ave., Ottawa, ON K1Y4E9, Canada

\section{J. Chow}

Hedley Orthopaedic Institute, 2122 E. Highland Ave., Ste 300, Phoenix, AZ 85016, USA

K. D. Olsen

Park Nicollet Clinic, 14000 Fairview Dr., Burnsville, MN 55337, USA

D. A. Fitch $(\bowtie)$

MicroPort Orthopedics Inc., 5677 Airline Rd., Arlington, TN 38002, USA

e-mail: david.fitch@ortho.microport.com rehabilitation facilities. Complications included dislocation $(0.8 \%)$, periprosthetic fracture $(0.8 \%)$, and deep vein thrombosis $(0.2 \%)$. There were no infections reported.

Conclusions Patients implanted using this tissue-sparing technique experienced reduced 30-day all-cause readmission rates ( $2.3 \%$ vs. $4.2 \%)$ and more were routinely discharged home (91.5\% vs. $27.3 \%$ ) than have been previously reported for patients in the United States. Use of this tissue-sparing technique has the potential to significantly reduce post-discharge costs.

Keywords Total hip arthroplasty · Tissue-sparing · Discharge status $\cdot 30$-day readmission rate $\cdot$ SuperPath

\section{Introduction}

The passage of the Affordable Care Act in the United States has increased attention on healthcare costs and led to the proposal of several value-based payment strategies. One example is a bundled payment option where all activities performed for an episode-of-care are reimbursed in a single fixed payment. The episode-of-care includes an anchor event, in this instance the total hip arthroplasty (THA) procedure, and a window of at least 30 days following discharge. Under this model, no additional compensation is provided for complications or unplanned readmissions occurring within the window. Bozic et al. recently reviewed payments for all total joint replacements (TJRs) performed at a single institution to examine the financial impact of bundling with this model [1]. They found $37 \%$ of the costs incurred for primary TJRs coded MS-DRG 470 were attributable to post-discharge activities, and that over $80 \%$ of those costs were attributable to unplanned readmissions and discharging patients to post-acute care facilities. 
One aspect of THA with the potential to impact both readmission rates and discharge status is the surgical technique. The supercapsular percutaneously-assisted total hip (SuperPath) surgical technique is a tissue-sparing approach that does not require specialty tables or the forced dislocation of the femoral head. The technique utilizes the interval between the gluteus medius and the piriformis to access the capsule without cutting any tendons or muscles [2]. It also allows for broaching directly through the femoral neck with the femoral head intact making it easier to replicate the natural femoral version and length. The resulting minimization of soft tissue damage allows for sameday ambulation and no standard postoperative patient restrictions. Initial reports with this surgical technique have found a mean hospital stay of 1.7 days with satisfactory radiographic outcomes and low complication rates [2].

The primary objective of this multicenter, retrospective study was to determine the 30-day all-cause readmission rate and discharge status for patients implanted using this surgical technique. The hypothesis is that these values will be improved compared to those previously reported in the United States and could in turn lead to potential post-discharge cost reductions.

\section{Methods}

The healthcare databases at three institutions were searched for all primary THAs performed by three surgeons who use the SuperPath surgical technique. The searches covered the period of January 2013 through July 2014. All primary THAs, regardless of indication or patient demographics, were included in the analysis. Collected data elements included the number of THAs, 30-day all-cause readmission rate, discharge status, transfusion rate, complications, and length of stay (LOS).

The 30-day all-cause readmission rate was defined as the percentage of patients who had a subsequent hospital admission in the same or a different hospital within 30 days of their THA procedure for any reason. Discharge status indicated the disposition of the patient at discharge from the hospital (e.g. home, skilled nursing facility). Transfusion rate was defined as the percentage of patients requiring a transfusion of any kind. Complication rate was defined as the percentage of patients experiencing each complication type (e.g. infection, dislocation). LOS was defined as the number of nights the patient remained in the hospital. A patient admitted and discharged on the same day had a LOS of zero days.

\section{Results}

Data were available for 479 THAs (Table 1). Readmission rates were similar at each site and $95.3 \%$ of patients were discharged home $(91.5 \%$ routinely and $3.8 \%$ to home health care). The combined transfusion rate was $3.3 \%$ and the mean LOS was 1.6 days. In total there were nine complications: four dislocations, four fractures, and one deep vein thrombosis (DVT). There were no reports of infection or pulmonary embolism.

\section{Discussion}

The present study reports the 30-day all-cause readmission rate and patient discharge status for patients undergoing THA with a tissue-sparing surgical technique. These two variables have been shown to contribute up to $81 \%$ of post-discharge costs within the first 30 days following TJR [1].

\section{Thirty-day readmission rate}

The American College of Surgeons National Surgical Quality Improvement Program (ACS NSQIP) database collects readmission and complication data from hospitals throughout the United States. Unlike Medicare claims data, this database has the benefit of providing information on patients of all ages. A recent review of this database reported the 30-day all-cause readmission rate to be $4.2 \%$ for over 8,100 THAs performed in 2011 [3].

The 30-day all-cause readmission rate from the present study was $2.3 \%$ (range, $1.5-3.2 \%$ ) or a $1.9 \%$ (range, 1.0 $2.7 \%$ ) reduction from the described national average. The lower readmission rate could be due to a reduction in key complications following THA. A separate review of the ACS NSQIP reported THA patients with surgical site infections, thromboembolic events, or sepsis were at an increased risk for readmission [4]. In the present study, there were no instances of infection or sepsis and only a single thromboembolic event $(0.2 \%)$. The lack of these complications could be due to several features of the SuperPath technique including: a reduced need for soft tissue dissection with electrocautery; a lack of need to cut muscles or tendons; and the reduced need for transfusions, which have been previously linked to an increased incidence of surgical site infections [5]. The dislocation rate $(0.8 \%)$ was also decreased from those reported in some recent THA studies (2.9-6.0\%) and could help to further explain the reduced readmission rate [6-8].

As described previously, unplanned readmissions within 30 days of a THA procedure are not reimbursable under certain bundled payment models and represent approximately $11 \%$ of post-discharge costs [1]. The economic burden of unplanned readmissions under this model is further illustrated by Bosco et al., who reported the direct costs to be $4.3 \%$ of reimbursement their hospital received for all THAs [9]. Or stated another way, hospital margins would need to be $4.3 \%$ 
Table 1 Outcomes from the three institutions presented separately and combined

\begin{tabular}{|c|c|c|c|c|}
\hline Parameter & Site 1 & Site 2 & Site 3 & Composite \\
\hline Number of THAs & 153 & 261 & 65 & 479 \\
\hline 30-Day readmission rate $(\%)$ & $3.2 \%$ & $2.0 \%$ & $1.5 \%$ & $2.3 \%$ \\
\hline \multicolumn{5}{|l|}{ Discharge status } \\
\hline Home $(\%)$ & $95.0 \%$ & $89.3 \%$ & $92.3 \%$ & $91.5 \%$ \\
\hline SNF (\%) & $5.0 \%$ & $3.0 \%$ & $6.1 \%$ & $4.1 \%$ \\
\hline HHC (\%) & - & $7.0 \%$ & - & $3.8 \%$ \\
\hline IRF (\%) & - & $0.7 \%$ & $1.5 \%$ & $0.6 \%$ \\
\hline Transfusion rate $(\%)$ & $8.0 \%$ & $0.7 \%$ & $3.0 \%$ & $3.3 \%$ \\
\hline Mean LOS (days) & 2.0 & 1.4 & 2.1 & 1.6 \\
\hline \multicolumn{5}{|l|}{ Complications } \\
\hline Dislocation (\%) & $1(0.06 \%)$ & $2(0.76 \%)$ & $1(1.5 \%)$ & $4(0.8 \%)$ \\
\hline DVT $(\%)$ & - & $1(0.38 \%)$ & - & $1(0.2 \%)$ \\
\hline Fracture $(\%)$ & - & $3(1.14 \%)$ & $1(1.5 \%)$ & $4(0.8 \%)$ \\
\hline Infection $(\%)$ & - & - & - & $0(0.0 \%)$ \\
\hline PE (\%) & - & - & - & $0(0.0 \%)$ \\
\hline
\end{tabular}

THA total hip arthroplasty, $S N F$ skilled nursing facility, $H H C$ home health care, $I R F$ inpatient rehabilitation facility, $L O S$ length of stay, $D V T$ deep vein thrombosis, $P E$ pulmonary embolism

greater than the reimbursement for all primary THA procedures to cover the cost of the non-reimbursed readmissions.

The potential to reduce readmission rates by $1.9 \%$ from the national average could translate to considerable cost savings for hospitals using the described bundled payment options. Bosco et al. estimated the direct cost of each unplanned readmission to be $\$ 17,103$ [9]. If an institution performed 100 THAs annually and reduced their 30-day readmission rate from 4.2 to $2.3 \%$, direct costs could be reduced by $45.2 \%$ or $\$ 32,496$ per year using Bosco's estimation (Table 2).

\section{Discharge status}

The Agency for Healthcare Research and Quality Healthcare Cost and Utilization Project (AHRQ HCUPnet) database was reviewed to determine the discharge status of all THA procedures (ICD-9-CM Code 81.51) performed in 2012 [10]. For over 299,000 THAs performed, $27.3 \%$ were discharged routinely home, $39.8 \%$ were discharged to home health care (HHC), $31.8 \%$ to another institution (nursing or rehabilitation facility), and $0.85 \%$ to another short-term hospital. In the present study, $91.5 \%$ of patients were discharged routinely home, $3.8 \%$ to $\mathrm{HHC}, 4.1 \%$ to a skilled nursing facility (SNF), and $0.6 \%$ to an inpatient rehabilitation facility (IRF). The cause for this increase in patients being discharged home is likely multifactorial, but could be related to the tissue-sparing nature of the subject surgical technique.

Discharging patients to post-acute care facilities accounts for $70 \%$ of post-discharge costs during the 30 days following TJR [1]. Ramos et al. examined Medicare claims data and determined the average costs were $\$ 16,464$ when discharging to an IRF, $\$ 6,678$ to a SNF, $\$ 4,239$ to $\mathrm{HHC}$, and $\$ 733$ for routine discharge home with outpatient therapy [11]. Using the example of an institution performing 100 THAs annually, there would be the potential to reduce costs by up $69.9 \%$ or $\$ 280,647$ if they were discharged according to the percentages found in the present study as opposed to those reported in the AHRQ HCUPnet database (Table 3). The standard AHRQ HCUPnet database report does not distinguish between SNF and IRF, so it is unclear what percentage of patients were discharged to each. To be most conservative in this example, it was assumed that all $31.8 \%$ were discharged to a SNF. If even $25 \%$ of these patients were actually sent to an IRF the potential cost reductions would increase to $\$ 358,446$ or $74.8 \%$.

\section{Other outcomes of interest}

Transfusion rates had the most variability between sites $(0.7-$ $8.0 \%$ ). This was not surprising as there are no consensus indications for transfusion and most centres have their own protocols. For instance, the protocol at site 1 was to transfuse patients when haemoglobin levels were lower than $8 \mathrm{~g} / \mathrm{dL}$, while all symptomatic patients with haemoglobin levels lower than $10 \mathrm{~g} / \mathrm{dL}$ were transfused at site 2 . Site 3 is a teaching hospital with junior residents who might be more likely to order transfusions when on-call.

Yoshihara et al. reviewed discharge data from the Nationwide Inpatient Sample database for TJRs performed between 2000 and 2009 to analyse transfusion trends in the United States [12]. They found the overall transfusion rate for THA 
Table 2 Example 30-day allcause readmission cost comparisons for the SuperPath procedure and values reported in the United States assuming a centre that performs 100 THAs annually

\begin{tabular}{|c|c|c|c|c|c|c|}
\hline \multirow[t]{2}{*}{$\begin{array}{l}\text { Direct cost per } \\
\text { readmission }(\$)\end{array}$} & \multicolumn{2}{|l|}{ Current study } & \multicolumn{2}{|c|}{$\begin{array}{l}\text { Previously reported US } \\
\text { values }\end{array}$} & \multicolumn{2}{|c|}{ Potential annual reductions } \\
\hline & $\begin{array}{l}\text { Readmission } \\
\text { rate }(\%)\end{array}$ & Cost (\$) & $\begin{array}{l}\text { Readmission } \\
\text { rate }(\%)\end{array}$ & Cost $(\$)$ & $\begin{array}{l}\text { Percent } \\
\text { difference (\%) }\end{array}$ & Cost (\$) \\
\hline$\$ 17,103[9]$ & $2.3 \%$ & $\$ 39,337$ & $4.2 \%[3]$ & $\$ 71,833$ & $45.2 \%$ & $\$ 32,496$ \\
\hline
\end{tabular}

patients was $25.5 \%$, which is similar to the $22.2 \%$ transfusion rate reported during a review of the ACS NSQIP [4]. The transfusion rate in the present study was $3.3 \%$. Minimizing transfusions is another key factor that could decrease the economic burden of THA, as Tuttle et al. estimated the cost of each transfusion to be $\$ 787.37$ at their institution [13]. In addition to directly adding cost, transfusions have also been linked to increased incidence of complications, including deep surgical site infections [5]. Therefore by minimizing transfusions, there is also the potential to reduce other complications that could result in readmissions.

The mean LOS in the present study was half the national average described in the AHRQ HCUPnet database (1.6 vs. 3.3 days) [10]. This reduction occurred along with a nearly $50 \%$ reduction in readmission rates, suggesting that the SuperPath surgical technique can result in more patients returning home in a shorter amount of time without compromising safety or increasing complication rates, at least within the first 30 days. The financial benefit of reduced LOS is more difficult to directly quantify, but higher patient turnover potentially allows for more procedures to be performed or the use of beds for other surgical procedures.

Also of interest is that the collected data from one site (site 3) represents outcomes associated with the surgeon's first cases performed using this technique or learning curve. Previous studies have shown that perioperative complications are higher during this phase for other surgical techniques [14, 15$]$. Despite this, outcomes for this site were similar to those reported at the other sites and lower than values previously reported in the United States. This data suggests the possibility of a minimal learning curve for this technique, at least in terms of perioperative outcomes and complications.

\section{Limitations}

There are several limitations to the present study. One site contributed over half of the included THAs, although results were similar across sites. The analysis was based upon an episode of care with a 30-day window following the procedure. There are numerous bundling models with varying window lengths and it is possible findings would differ by looking at longer windows. The provided example calculations are based upon previously published values instead of analysis of actual costs incurred by the sites. Finally, there are numerous factors that could improve the variables analysed in this study outside of surgical technique alone such as early ambulation protocols.

\section{Conclusions}

In conclusion, the SuperPath surgical technique was associated with a decreased 30-day all-cause readmission rate and fewer patients were discharged to post-acute care facilities. Example calculations show the potential for combined cost reductions of up to $66.2 \%$ for these two variables alone, which as a previous study reported account for $81 \%$ of post-discharge costs overall. Over $95 \%$ of patients in the present study were discharged home $(91.5 \%$ routinely and $3.8 \%$ to $\mathrm{HHC}$ ), and there were no instances of infection in nearly 500 cases. These results show use of this technique has the potential to significantly reduce post-discharge costs associated with THA. Future prospective studies examining the actual costs incurred by hospitals are needed to confirm the

Table 3 Example discharge status cost comparisons for the SuperPath procedure and values reported in the United States assuming a centre that implants 100 THAs annually

\begin{tabular}{|c|c|c|c|c|c|c|c|}
\hline \multirow{2}{*}{$\frac{\text { Discharge status }}{\text { Home }(\%)}$} & \multirow{2}{*}{$\begin{array}{l}\text { Average cost per discharge (\$) } \\
\$ 733.00[11]\end{array}$} & \multicolumn{2}{|c|}{ Present study } & \multicolumn{2}{|c|}{ Previously reported US values } & \multicolumn{2}{|c|}{ Potential annual reductions } \\
\hline & & $91.5 \%$ & $\$ 67,069.50$ & $27.3 \%[10]$ & $\$ 20,010.90$ & - & - \\
\hline SNF $(\%)$ & $\$ 6,678.00[11]$ & $4.1 \%$ & $\$ 27,379.80$ & $31.8 \%[10]^{\mathrm{a}}$ & $\$ 212,360.40$ & - & - \\
\hline HHC (\%) & $\$ 4,239.00[11]$ & $3.8 \%$ & $\$ 16,108.20$ & $39.8 \%[10]$ & $\$ 168,712.20$ & - & - \\
\hline $\operatorname{IRF}(\%)$ & $\$ 16,464.00[11]$ & $0.6 \%$ & $\$ 9,878.40$ & $0.0 \%$ & $\$ 0$ & - & - \\
\hline Total & - & - & $\$ 120,435.90$ & - & $\$ 401,083.50$ & $69.9 \%$ & $\$ 280,647.60$ \\
\hline
\end{tabular}

$I R F$ inpatient rehabilitation facility, $S N F$ skilled nursing facility, $H H C$ home health care

${ }^{a}$ As stated in text, it was assumed all $31.8 \%$ were discharged to SNFs 
improved variables described in this study actually result in cost reductions.

Open Access This article is distributed under the terms of the Creative Commons Attribution License which permits any use, distribution, and reproduction in any medium, provided the original author(s) and the source are credited.

\section{References}

1. Bozic KJ, Ward L, Vail TP, Maze M (2014) Bundled payments in total joint arthroplasty: targeting opportunities for quality improvement and cost reduction. Clin Orthop Relat Res 472(1):188-193. doi: 10.1007/s11999-013-3034-3

2. Chow J, Penenberg B, Murphy S (2011) Modified micro-superior percutaneously-assisted total hip: early experiences \& case reports. Curr Rev Musculoskelet Med 4(3):146-150. doi:10.1007/s12178011-9090-y

3. Pugely AJ, Callaghan JJ, Martin CT, Cram P, Gao Y (2013) Incidence of and risk factors for 30-day readmission following elective primary total joint arthroplasty: analysis from the ACS-NSQIP. J Arthroplast 28(9):1499-1504. doi:10.1016/j.arth.2013.06.032

4. Mednick RE, Alvi HM, Krishnan V, Lovecchio F, Manning DW (2014) Factors affecting readmission rates following primary total Hip arthroplasty. J Bone Joint Surg Am Vol 96(14):1201-1209. doi: 10.2106/JBJS.M.00556

5. Frisch NB, Wessell NM, Charters MA, Yu S, Jeffries JJ, Silverton CD (2014) Predictors and complications of blood transfusion in total hip and knee arthroplasty. J Arthroplast. doi:10.1016/j.arth.2014.03.048

6. Itokawa T, Nakashima Y, Yamamoto T, Motomura G, Ohishi M, Hamai S, Akiyama M, Hirata M, Hara D, Iwamoto Y (2013) Late dislocation is associated with recurrence after total hip arthroplasty. Int Orthop 37(8):1457-1463. doi:10.1007/s00264-013-1921-6

7. Swanson TV (2005) Early results of 1000 consecutive, posterior, single-incision minimally-invasive surgery total hip arthroplasties. J Arthroplast 20(Suppl 3):26-32. doi:10.1016/j.arth.2005.05.017

8. Chevillotte C, Pibarot V, Carret J, Bejui-Hugues J, Guyen O (2011) Nine years follow-up of 100 ceramic-on-ceramic total hip arthroplasty. Int Orthop 35(11):1599-1604. doi:10.1007/s00264010-1185-3

9. Bosco JA 3rd, Karkenny AJ, Hutzler LH, Slover JD, Iorio R (2014) Cost burden of 30-day readmissions following medicare total hip and knee arthroplasty. J Arthroplast 29(5):903-905. doi:10.1016/j.arth. 2013.11.006

10. AHRQ HCUPnet (2012) Agency for Healthcare Research and Quality H-CUPnet Database, ICD-9-CM Code 81.51 for United States in 2011. http://hcupnet.ahrq.gov/HCUPnet.jsp. Accessed 4 Sept 2014

11. Ramos NL, Wang EL, Karia RJ, Hutzler LH, Lajam CM, Bosco JA 3rd (2014) Correlation between physician specific discharge costs, LOS, and 30-day readmission rates: an analysis of 1,831 cases. J Arthroplast 29(9):1717-1722. doi:10.1016/j.arth.2014. 04.005

12. Yoshihara H, Yoneoka D (2014) National trends in the utilization of blood transfusions in total hip and knee arthroplasty. J Arthroplast 29(10):1932-1937. doi:10.1016/j.arth.2014.04.029

13. Tuttle JR, Ritterman SA, Cassidy DB, Anazonwu WA, Froehlich JA, Rubin LE (2014) Cost benefit analysis of topical tranexamic acid in primary total hip and knee arthroplasty. J Arthroplast 29(8):15121515. doi:10.1016/j.arth.2014.01.031

14. Bal BS, Haltorn D, Aleto T, Barrett M (2005) Early complications of primary total hip replacement performed with a two-incision minimally invasive technique. J Bone Joint Surg Am 87(11):2432-2438. doi:10.2106/JBJS.D.02847

15. Yi C, Agudelo JF, Dayton MR, Morgan SJ (2013) Early complications of anterior supine intermuscular total hip arthroplasty. Orthop 36(3):e276-e281. doi:10.3928/01477447-20130222-14 